\title{
Probiotics (Lactobacillus acidophilus and Bifidobacterium bifidum) prevent NEC in VLBW infants fed breast milk but not formula
}

Andreas Repa, Margarita Thanhaeuser, David Endress, Michael Weber, Alexandra Kreissl, Christoph Binder, Angelika Berger and Nadja Haiden

Pediatric Research (2015) 77, 381-388 doi:10.1038/pr.2014.192

The originally published title of this article is incorrect. The correct title is "Probiotics (Lactobacillus acidophilus and Bifidobacterium infantis) prevent NEC in VLBW infants fed breast milk but not formula." The authors regret the error. 